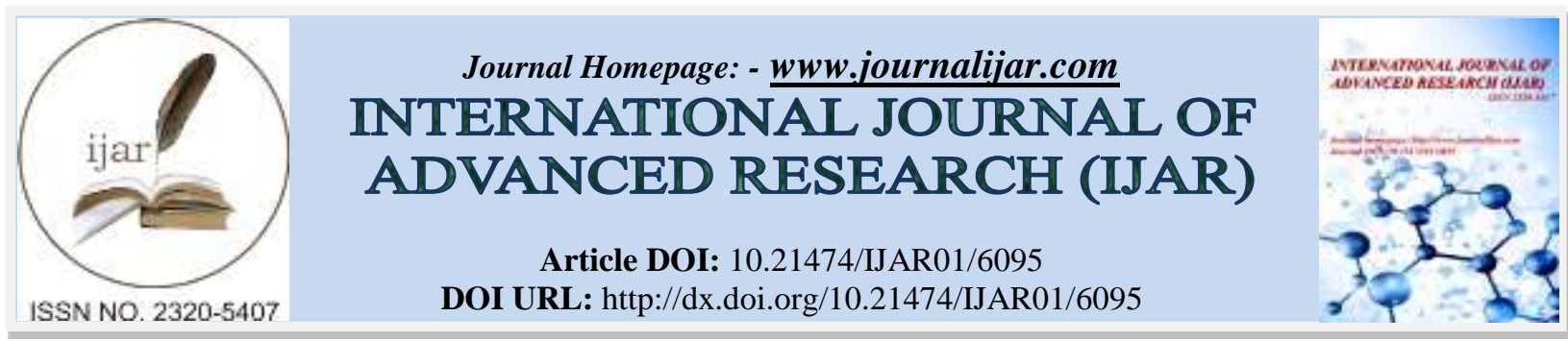

RESEARCH ARTICLE

\title{
INFLUENCE OF VERTICAL JUMP PERFORMANCE ON ACCELERATION, MAXIMAL SPEED AND CHANGE OF DIRECTION SPEED IN PROFESSIONAL SOCCER PLAYERS.
}

\author{
Bahar Ateş ${ }^{1}$ and Ebru Çetin ${ }^{2}$. \\ 1. University of Uşak, Faculty of Sport Sciences, Uşak, Turkey. \\ 2. University of Gazi, Faculty of Sport Sciences, Ankara, Turkey.
}

\section{Manuscript Info}

Manuscript History

Received: 18 October 2017

Final Accepted: 18 November 2017

Published: December 2017

Keywords:-

Power, agility, linear speed, field tests.

\begin{abstract}
In this study, we evaluated the efficacy of vertical jump performance on acceleration, maximal speed and change of direction speed in professional soccer players. A total of 18 football players, mean age: $24.6 \pm 2.7$ years, mean height: $1.80 \pm 0.06 \mathrm{~cm}$, mean weight: $75.4 \pm 5.75$ $\mathrm{kg}$ and mean body fat percentage $11.8 \pm 1.38 \%$, in a 3rd Division Professional Soccer Team were voluntarily participated in this study. At the completion of the warm-up protocol, players completed assessments of countermovement jump (CMJ), speed (10-, and 30-m sprints) and change of direction speed (Pro Agility and T-test) tests. Spearman correlation analysis was used to identify the relationships between the CMJ and speed tests. Players displayed a significant negative moderate correlations between CMJ and $10-\mathrm{m}(\mathrm{r}=-0.59 ; \mathrm{p}=$ $0.01)$, and CMJ and $30-\mathrm{m}(\mathrm{r}=-0.63 ; \mathrm{p}=0.00)$. Also it was found significant negative strong correlation between CMJ and T-test $(\mathrm{r}=$ $0.81 ; \mathrm{p}=0.00$ ). However, no significant correlation was found between CMJ and Pro Agility Test $(r=-0.22 ; p=0.19)$. The results of this study show that there is a moderate-strong relationship between power and speed ability in soccer players.
\end{abstract}

Copy Right, IJAR, 2017,. All rights reserved.

\section{Introduction:-}

Soccer is perceived as an aerobic endurance sport (Pinasco and Carson, 2005) because the duration of a soccer game is long (about 90 minutes) and the athlete covers a distance of over $10 \mathrm{~km}$ during the game (Bangsbo et al., 2006). However, in recent years, football has become a very fast-paced game (Wallace et al., 2014) and the emphasis on changing low and high violent leaps, sprints, tactics, and many shifting movements (Hammami et al. 2017) has led to the examination of high-intensity activities in soccer for the last two decades (Carling et al., 2012).

During the game, the players perform about 1300 changes of direction (Stølen et al., 2005; Vigne et al., 2010) involving 220 high-intensity movements at a speed of 2-4 seconds every 90 seconds (McFarland et al. 2016). A professional soccer player must have the ability to maintain these movements at high speed and high pace during the entire game (Little and Williams, 2005). In other words, agility/change of direction team sports such as soccer, rugby, American football and ice hockey (Lockie et al., 2003) and numerous linear sprints ranging from 1.5 to 150 meters are shown as essential qualities that determine performance (McFarland et al. 2016) Regarding these changes, training programs have also been directed at improving sprint, repeated sprint, change of direction, strength and power characteristics of aerobic adaptation (Bedoya, 2015; Enright et al. 2015; Ramirez-Campillo et al. 2015; 
Wisloff et al. 2004). Furthermore, these movements are often used to assess neuromuscular performance in soccer (Arcos et al. 2017).

In soccer, the explosive force measured as a vertical jump is considered to be beneficial in achieving optimal performance, regarding determining talent and in evaluating physical performance (Stølen et al., 2005). For example, vertical jump performance comparisons measured as countermovement jump (CMJ) and squat jump (SJ) in team sports are frequently used to assess lower leg stretch-shortening cycle activity (McGuigan et al., 2006). The vertical jump test does not directly measure power performance, but it is applied by coaches in most sports branches (Farland et al., 2016) because it is an easy and cost-effective field assessment due to the strength of the jump being a measurable result of power generation. Many studies have reported that explosive power performance, determined by vertical jump tests such as CMJ and SJ, is associated with acceleration, maximal speed, and the ability to change of direction (Little and Williams, 2005; Lockie et al, 2014; Loturco Lockie et al., 2015; Cronin and Hansen, 2005). There are also studies reporting that leg strength is a decisive factor regarding rate of change of direction in young and professional soccer players (Lahance et al., 2009). However, when the studies are examined, consistent results cannot be obtained. While Fatih (2009) found a moderate relationship between vertical jump performance and change of direction speeds in girls and boys, Nimphius et al. (2010) in their study, could not determine a significant relationship between vertical jump height and performance measurements.

To date, the relationship between these characteristics of athletic performance, such as sprinting, agility/ change of direction which are used as physical fitness measures for soccer, remains uncertain, despite the fact of the presence of many studies in term of the relationship between sprint, jumping and change of direction. In this context, the aim was to investigate the effect of CMJ on acceleration, maximal speed and change of direction ability of male soccer players playing in a professional 3rd division football team.

\section{Methodology:- \\ Participants:-}

18 athletes participating in a 3rd Division Professional Soccer Team, with a mean age of $24.6 \pm 2.7$ years, mean height of $1.80 \pm 0.06 \mathrm{~cm}$, mean weight of $75.4 \pm 5.75 \mathrm{~kg}$ and mean body fat percentage of $11.8 \pm 1.38 \%$, voluntarily participated in this study. At the beginning of the study, participant athletes were informed about research protocols. The study was carried out by the principles of the Helsinki Declaration. The assessment. Informed written consents were obtained from the athletes prior to participation in the study. The study was approved by the Health Science Ethics Committee (approval no: 2016-36). In addition, all experimental procedures were conducted in accordance with the Declaration of Helsinki.

\section{Protocol:-}

All tests were carried out in a single session before the 2017/2018 season. Before the tests, the athletes were asked to wear clothes and shoes that would not restrict their movements. All athletes were given exercises during the week before the study to familiarize themselves with the tests. Athletes were informed that they should not perform any intense activity 48 hours before the measurements. The order of testing was designed in such a way that one test would not adversely affect the performance of another test, their order being as follows; jump test, agility tests and sprint measurements. After the anthropometric measurements were measured and recorded for each athlete, a standard warm-up was performed by the coach before the test. This warm-up lasted about 10 minutes and consisted of light walking, running and dynamic stretching exercises. Subsequently, participants were allowed to perform three sub-maximal trials for each test. First, the countermovement jump (CMJ) test was performed. Immediately after this measurement, the athletes performed Pro-agility and T-test agility tests. After the agility tests were performed, 10-m and 30-m sprint tests were performed on the grass field. The test was repeated three times for each participant regarding establishing reliability for the test and the best value was recorded (Hopkins, 2000).

\section{Anthropometric Data:-}

The athlete's heights were measured with a scale having a sensitivity of $0.01 \mathrm{~m}(\mathrm{~m})$, and their body weights (VA) were measured with electronic scales (SECA, Germany) with a sensitivity level of 0.1 kilograms (kg). Skin fold thickness values were determined in seven areas (triceps, supra-iliac, subscapular, calf, thigh, pectoral, abdominal) with a skinfold caliper (Holtain, UK) applying a pressure of $10 \mathrm{gr}$ to $1 \mathrm{~mm} 2$ with a $\pm 2 \mathrm{~mm}$ error rate. 


\section{Countermovement Jump (CMJ) Test:-}

The CMJ performance of the athletes was measured by Smart jump (Fusion Sport, Australia). All the athletes were asked to sit on a mat, their hands on their buttocks and to jump as high as possible when ready. The athletes, their hands on their hips and in an upright stance, rapidly collapsed downward and jumped to the maximum attainable height without pulling their knees upward while maintaining stretched legs. Resting intervals of $\leq 1$ min were applied to reduce possible tiredness. The time indicator started to work with the accuracy of 0.001 seconds at the moment the participants were on the jumping platform, and the contact of their feet with the floor was lost. The time was stopped when the participant landed back on the platform, and the airborne duration of the participant was determined. Obtained jump heights were evaluated in $\mathrm{cm}$. Before starting the jump test, the participant was asked to look at a fixed spot in front of them so that they could land on the same spot of the mat as often as possible during the jump.

\section{Pro-Agility Test:-}

During the Pro-Agility Tests (Fig. 1) the time was measured by using a hand-held chronometer, by rounding to nearest $0.10 \mathrm{sec}$. The participants placed themselves on the starting line before the test. When ready, they first touched the mark on the right and then the mark on the left and finished the test by passing through the starting line and running rapidly, when their foot touched the determined line the test was concluded, in this exercise, the participant performed a maximum of 3 repetitions with full rests in-between.

\section{T-Test:-}

To prepare the track, the four cones were arranged on the track as shown in Fig.2 When the participant receives the start command, he starts from the "A" cone, runs straight to the "B" cone and touches the cone with the right hand. Then he runs to the left side to the " $\mathrm{C}$ " cone in side step and touches the cone with the left hand, then he runs to the right side step and touches the " $\mathrm{D}$ " cone with the right hand. Then he runs side step back to the " $\mathrm{B}$ " cone and touches the cone with the left hand, and thereafter he runs back to the "A" cone. As soon as he reaches the "A" cone, the stopwatch is stopped. In this measurement, the participant made a maximum of 3 repetitions with full rests inbetween.

\section{Sprint Tests:-}

Sprint speed (10-m and 30-m) was measured on the grass scene with a photocell stopwatch system. When $10 \mathrm{~m}$ was given the acceleration criterion, it was evaluated as the maximal sprint velocity measured at $30-\mathrm{m}$. In these tests, the photocell doors were placed at the start and finish distances. Footballers started from a standing position $0.3 \mathrm{~m}$ ahead of the starting line. Each athlete was given three trials every 2 minutes, and the best rating was used.

\section{Statistical Analysis:-}

Statistical analysis of the values obtained from the study was performed using the IBM SPSS (version 23) analysis program. First, the arithmetic mean, standard deviation values of the data were calculated. The values of skewness and kurtosis were checked to determine whether the data were appropriate for normal distribution. Spearman Correlation Analysis was performed to determine the relationship between countermovement jump (CMJ), acceleration, maximal velocity, and agility test time variables. The $95 \%$ confidence interval for each variable was calculated. The significance level for all calculations was interpreted as $\mathrm{p}<0.05$, R-values were evaluated as weak $(\leq 0.39)$, moderate $(0.40 \leq \mathrm{r} \leq 0.69)$ or strong $(\geq 0.70)$ (Cohen et al. 2013).

\section{Results:-}

The players' test performances are shown in Table 1. CMJ ability showed moderate negative correlation with both 10-m and 30-m sprint (Figure 1,2) and a strong negative correlation with the T-test agility performance (Figure 3). In contrast, no significant correlation was found between CMJ ability and pro-agility shuttle. In addition, almost a strong positive correlation was detected between $30-\mathrm{m}$ sprint times and both the pro-agility shuttle and T-test. Athletes showed moderate to strong correlations between 10-m sprint times and T-test and $30-\mathrm{m}$ sprint times. There was a moderate correlation between pro-agility shuttle and T-test performance (Table 2). 
Table 1:- Performance data (mean \pm standard deviation (SD); 95\% confidence intervals [CI])

\begin{tabular}{|l|c|c|c|}
\hline \multicolumn{1}{|c|}{ Performance Tests } & $\mathrm{N}$ & Mean $\pm \mathrm{SD}$ & $95 \%$ Confidence Intervals (CI) \\
\hline Acceleration & 18 & $1.64 \pm 0.06$ & $1.61-1.67$ \\
\hline $10 \mathrm{~m}$ & 18 & $4.08 \pm 0.13$ & $4.01-4.15$ \\
\hline Maximal Velocity & $5.81-6.18$ \\
\hline $30 \mathrm{~m}$ & 18 & $6.11 \pm 0.41$ & $9.67-10.1$ \\
\hline CODS & 18 & $9.91 \pm 0.51$ & $37.9-39.8$ \\
\hline Pro Agility (s) & 18 & $38.8 \pm 1.85$ & \\
\hline T-test (s) &
\end{tabular}

CMJ: counter movement jump

Table 2:- Correlations between CMJ, Acceleration, Maximal Velocity and CODS (r-values)

\begin{tabular}{|l|c|c|c|c|}
\hline & $\mathbf{1 0} \mathbf{~ m}$ & $\mathbf{3 0 ~}$ & Pro Agility Test & T-Test \\
\hline $\mathbf{3 0} \mathbf{~ m}$ & $0.73 * *$ & - & & \\
\hline Pro Agility & 0.22 & $0.69 * *$ & - & \\
\hline T-test & $0.61 * *$ & $0.69 * *$ & $0.51 * *$ & - \\
\hline CMJ & $-0.59 * *$ & $-0.63 *$ & -0.22 & $-0.81 * *$ \\
\hline
\end{tabular}

$* \mathrm{p}<0.05 ; * * \mathrm{p}<0.01 \quad$ CMJ: Counter movement jump

\section{Discussion:-}

In team sports, athletes need strength and power to run, jump, deflect, attack, and fight. For this reason, in many studies, the relationship between countermovement jump (CMJ) and squat jump (SJ) tests, which are forms of vertical jumping and sprint and direction changing abilities is being investigated (McFarland et al., 2016; Arcos ve ark., 2017; Braz et al., 2017; Meylan et al., 2009). From this point of view, this study aims to examine the effect of power production on professional soccer players regarding sprint and change of direction abilities. At the end of the study, it was determined that there is a moderate-strong relationship between speed and power ability.

A significant negative correlation was present between CMJ and 10-m and 30-m sprint values these being respectively $(r=-0.59 ; \mathrm{p}=0.01)$ and $(\mathrm{r}=-0.63 ; \mathrm{p}=0.00)$. Young et al. (2002) found statistically significant results between reactive force, regarding jumping, flat sprint and sprint in different directions in a study related to soccer, basketball, Australian soccer and tennis athletes, studying the relationship between falling and leaping and $8 \mathrm{~m}$ sprint performances in straight and different directions. Volpi Braz et al. (2017) found a significant relationship between the two vertical bounces (CMJ and SJ) and the relationship between sprint velocities and variables. At the end of the study, a significant negative correlation $(r=-0.69, \mathrm{p}<0.05)$ was noted between CMJ and $10 \mathrm{~m}$ sprint. Köklü et al. $(\mathrm{R}=-0.123)$ found that there was no significant correlation between the CMJ ability and the 10-meter sprinting rate in the young football players who played at the same time (2013), a moderate-strong significant correlation $(r=-0.599, \mathrm{p}<0.05)$. A stronger correlation $(27.4$ and $36.6 \mathrm{~m})$ was found in college and high school women, and it was determined that in longer distances (27.4 and $36.6 \mathrm{~m}$ ) with CMJ (Vescovi and McGuigan, 2008) was observed in short distances $(9.1$ and $18.3 \mathrm{~m})$. The findings are in parallel with the literature. Coaches can use the CMJ test, especially when evaluating maximal speed when they are evaluating soccer players.

Other findings in the current study indicate that a strong negative correlation $(\mathrm{r}=-0.81 ; \mathrm{p}=0.00)$ was found between the CMJ and T-test performance scores, while there was a significant correlation between the CMJ ability and the Pro agility test $(r=-0.22 ; p=0.19)$. A similar study was performed by McFarland et al. (2016) on women and men playing in the 2 nd league university soccer. In a study of the relationship between two different jumping tests (active and squat jumping) and sprint and directional change velocity abilities, a strong negative correlation $(\mathrm{r}=-0.76$, $\mathrm{p} \leq 0.05$ ) was observed between $\mathrm{CMJ}$ and $\mathrm{T}$-Test only in female soccer players. There was no meaningful relationship between CMJ and agility tests in male soccer players. In another study by Volpi Braz et al. (2017), it was reported that there was a strong correlation $(\mathrm{r}=-0.53, \mathrm{p}<0.05)$ between the CMJ and the Zig Zag Test in the study of Brazilian elite soccer players. Depending on other factors (such as gender, competition level, age), different types of jumping, sprinting tests at different distances and different agility tests make it difficult to compare these agility tests with unilateral or bilateral use of studies on different ground applications. For example, in a study of male team athletes evaluating vertical jump performance unilaterally and bilaterally, a significant correlation was 
observed in the right leg $(r=-0.380$ to $-0.512, p \leq 0.05)$, although there was no significant correlation between left leg vertical jump performance. In the same study, a significant correlation was found between the left leg long jump and lateral jump with both T-test and 505 agility tests $(r=-0.370$ to $-0.729, p \leq 0.05)$ (Lockie et al. 2014). Mujika et al. (2009) reported that CMJ performance varied regarding sex and competition level in professional women's teams. Arcos et al. (2017) analyzed the leap, acceleration and directional changes in motor skills in 42 male soccer players in the 2nd and 3rd leagues. Vertical jumping, squat jumping, active jumping and accelerating together with active jumping with free arms, 5-10-15 m sprint and direction change tests were conducted with a changeable free agility test (MATF), 505 agility test (505) and 20-yard agility test (Y20). At the end of the study, it has been stated the ability to change direction with acceleration is an indication of a very large correlation between sprint performance and sprint tests, these being $(r=.26-.53)(r=.-33-.-64)$, respectively.

\section{Conclusion:-}

As a result, this study shows that power has positive effects on the speed ability of professional soccer players. Sprinting abilities can be improved on soccer players by incorporating stretching-shortening cycling exercises, which improve the ability to jump, into training programs. However, when the literature is examined, it is understood that jumping, acceleration, and change of direction abilities are independent of each other in a soccer player. For this reason, it is recommended that these skills be developed and evaluated separately.

As with other studies, this study has its limitations. Since only male professional 3rd division soccer players were included in the study, the results of this study do not apply to the entire soccer playing population. Future studies should also consider different levels of competition, age groups, gender and game positions when examining the relationship between strength and speed.

\section{Acknowledgement:-}

The authors would like to thank the soccer team for their participation and support of this study.

\section{References:-}

1. Bangsbo, J., Mohr, M., Krustrup, P. (2006): Physical and metabolic demands of training and match-play in the elite football player. Journal of Sports Science, 24: 665- 674.

2. Bedoya, A.A. (2015): Plyometric Training Effects on Athletic Performance in Youth Soccer Athletes: A Systematic Review. The Journal of Strength \& Conditioning Research, 29: 2351-2360.

3. Carling, C., Franck L.G., Gregory, D. (2012): Analysis of repeated high-intensity running performance in professional soccer." Journal of Sports Sciences, 30: 325-336.

4. Cohen, J., Cohen, P., West, S.G., Aiken, L.S. (2013): Applied Multiple Regression/Correlation Analysis for the Behavioral Sciences; Routledge: London, UK.

5. Cronin, J., Hansen, K. (2005): Strength and power predictors of sports speed Journal of Strength \& Conditioning Research, 19: 349-357.

6. Enright, K., Morton, J., Iga, J., Drust, B. (2015): The effect of concurrent training organisation in youth elite soccer players. European Journal of Appled Physiolgy, 115: 2367-2381.

7. Fatih, H. (2009). The relationship of jumping and agility performance in children. Science Moment Health, 9: $415-419$.

8. Hammami, M., Negra, Y., Shephard, R.J., Chelly, M.S. (2017): The Effect of Standard Strength vs. Contrast Strength Training on the Delopment of Sprint, Agility, Repeated Change of Direction, and Jump in Junior Male Soccer Players. The Journal of Strength \& Conditioning Research, 31: 901-912.

9. Hopkins, W.G. (2000): Measures of reliability in sports medicine and science. Sports Medicine, 30: 1-15.

10. Lehance, C., Binet, J., Bury, T., Croisier, J.L. (2009): Muscular strength, functional performances and injury risk in professional and junior elite soccer players. Scandinavian Journal of Medicine \& Science in Sports, 19: 243-251.

11. Little, T., Williams, A.G. (2005): Specificity of acceleration, maximum speed, and agility in professional soccer players. Journal of Strength and Conditioning Research, 19: 76-78.

12. Lockie, R.G., Murphy, A.J., Spinks, C.D. (2003): Effects of resisted sled towing on sprint kinematics in fieldsport athletes. Journal of Strength and Conditioning Research, 17: 760-767.

13. Los Arcos, A., Jurdan, M., Yanci, J. (2017): Specificity Of Jumping, Acceleration And Quick Change Of Direction Motor Abilities İn Soccer Players. Kinesiology 49: 22-29. 
14. Loturco, I., Pereira, L.A., Cal Abad, C.C., D'Angelo, R.A., Fernandes, V., Kitamura, K., et al. (2015): rtical and Horizontal Jump Tests Are Strongly Associated With Competiti Performance in 100-m Dash Ents. Journal of Strength \& Conditioning Research, 29: 1966-1971.

15. McFarland, I.T., Dawes, J.J., Elder, C.L., Lockie, G.R. (2016): Relationship of two rtical jumping tests to sprint and change of direction speed among male and female collegiate soccer players. Sports, 4: 1-7.

16. McGuigan, M.R., Doyle, T.L., Newton, M., Edwards, D.J., Nimphius, S., Newton, R.U. (2006): Eccentric utilization ratio: Effect of sport and phase of training. Journal of Strength \& Conditioning Research, 20: $992-$ 995.

17. Meylan, C., McMaster, T., Cronin, J., Mohammad, N.I., Rogers, C. (2009): Single-leg lateral, horizontal, and rtical jump assessment: reliability, interrelationships, and ability to predict sprint and change-of-direction performance. The Journal of Strength \& Conditioning Research, 23: 1140-1147.

18. Mujika, I., Santisteban, J., Impellizzeri, F.M., Castagna, C. (2009): Fitness determinants of success in men's and women's football. Journal of Sports Science, 27: 107-114.

19. Nimphius, S., McGuigan, M., Newton, R. (2010): Relationship between strength, power, speed, and change of direction performance in female softball players. Journal of Strength \& Conditioning Research, 24: 885-895.

20. Pinasco, A., Carson, J. (2005): Preseason conditioning for college soccer. The Journal of Strength and Conditioning Research, 27: 56-62.

21. Ramirez-Campillo, R., Gallardo, F., Henriquez-Olguin, C., Meylan, C.M., Martinez, C., Alvarez, C., et al. (2015): Effect of rtical, Horizontal, and Combined Plyometric Training on Explosi, Balance, and Endurance Performance of Young Soccer Players. The Journal of Strength \& Conditioning Research, 29: 1784-1795.

22. Stølen, T., Chamari, K., Castagna, C., Wisløff, U. (2005): Physiology of soccer: An update. Sports Medicine, 35: 501-536.

23. Scovi, J.D., Mcguigan, M.R. (2008): Relationships between sprinting, agility, and jump ability in female athletes. Journal of Sports Sciences, 26: 97-107.

24. Vigne, G., Gaudino, C., Rogowski, I., Alloatti, G., Hautier, C. (2010): Activity profile in elite Italian soccer team. International Journal of Sports Medicine, 31: 304-310.

25. Volpi Braz, T., José Nogueira, W., de Assis Cruz, W., Borsetti Businari, G., de Ornelas, F., Als Brigatto, F., et al. (2017): Relation between Different Variables of rtical Jumps and Sprints in Brazilian Professional Soccer Players. Journal of Exercise Physiology Online, 20: 33-46.

26. Wallace, J.L., Norton, K.I. (2014): Evolution of World Cup soccer final games 1966-2010: Game structure, speed and play patterns. Journal of Science and Medicine Sport, 17: 223-228.

27. Wisloff, U., Castagna, C., Helgerud, J., Jones, R., Hoff, J. (2004): Strong correlation of maximal squat strength with sprint performance and rtical jump height in elite soccer players. British Journal of Sports Medicine, 38 : 285-288.

28. Young, W.B., James, R., Montgomery, I. (2002): Is muscle power related to running speed with changed of direction?. Journal of Sports Medicine and Physical Fitness, 42: 282. 